(Aus dem tierphysiol. Institut der landw. Hochschule zu Berlin. Prof. Zuntz.)

\title{
Über \\ den Ersatz von Eiweiss durch Leim im Stoffwechsel.
}

Von

Dr. med. M. Kauffmanu.

In seiner akademischen Antrittsrede Zürich 1868 führte L. Hermann aus, dass sich sämtliche chemische Verdauungsprozesse als Spaltungen unter Wasseraufnahme auffassen lassen, dergestalt, dass die ganze chemische Verdauung sich ersetzen lassen würde durch Kochen der Nąhrung mit verdünnter Schwefelsäure.

Nach Otto Cohnheim ${ }^{1}$ ) geht die Verdaung so vor sich, dass „das von den höheren Tieren verzehrte Nahrungseiweiss im Magendarmkanal durch die proteolytischen Fermente Pepsin, Trypsin und Erepsin bis zu den primären Spaltungsprodukten, den Aminosäuren usw. gespalten und in dieser Form resorbiert wird".

Auf die Anregung Hermann's unternahm es Escher ${ }^{2}$ ), das Eiweiss in der Nahrung durch Leim und Tyrosin zu ersetzen. Er fütterte Schweine und Hunde mit Leim, dann mit Leim und Tyrosin und beobachtete, dass, während bei Leimnahrung allein das Körpergewicht abnahm, dieses bei Zugabe von Tyrosin gleichblieb. Er kombinierte Leim mit Tyrosin, weil diese aromatische Aminosäure weder bei hydrolytischer Spaltung noch bei Fäulnis im Leim nachgewiesen wurde.

K. B. Lehmann ${ }^{3}$ ) fütterte Ratten mit Leim und Tyrosin; alle Tiere gingen spätestens nach 70 Tagen zugrunde. Er schloss daraus, dass wenigstens Ratten die Eiweisssynthese aus Leim und Tyrosin nicht auszuführen vermögen. Vergleicht man damit die Versuche

1) Chemie der Eiweisskörper S. 55.

2) Über den Ersatz des Eiweisses durch Leim und Tyrosin. Vierteljahrsschrift der naturforschenden Gesellschaft in Zürich 1876 p. 36.

3) Sitzungsber. der Münchener morph.-physiol. Gesellsch. 10. März 1885. 
von Lunin ${ }^{11}$, dem es nicht gelang, mit Kasein, Zucker, Fett und Salzen Tiere dauernd am Leben zu erhalten, also mit Stoffen, die doch nach unserer bisherigen Annahme genügen sollten zur vollwertigen Ernährung, so scheint Lehmann's Schlussfolgerung zu weitgehend. Otto Loewi ${ }^{2}$ ) erklärt die Lunin'schen Versuche so, "dass ausser den Stickstoffträgern möglichst alle für den Aufbau eventuell in Betracht kommenden Elemente gereicht werden müssen. Was wir hierzu zu rechnen haben, ist unbekannt. In unseren gewöhnlichen Nahrungsmitteln, müssen wir annehmen, sind ausser Eiweiss, Fett, Kohlehydraten und Salzen, wenn auch nur in minimen Mengen, Stoffe enthaiten, die für den normalen Lebenslauf unersetzlich sind. Man denke nur an die bereits bekannte Bedeutung des Jods oder Eisens. Solange wir diese ,Minimum'stoffe nicht kennen, müssen wir bei allen Versuchen über künstliche Ernährung mit der Möglichkeit eines unkontrollierbaren Fehlers rechnen." Wenn also Lunin nicht einmal mit Eiweiss Tiere dauernd am Leben erhalten konnte, so ist erst recht nicht abzusehen, weshalb dies mit Leim und Tyrosin bätte glücken sollen. Es ist also eine Eiweisssynthese im Tierkörper mit letzteren Stoffen durch $L$ e h man n's Versuche nicht auszuschliessen.

Die Rolle des Eiweisses im Tierkörper ist eine doppelte; es dient als Energiequelle wie die N-freien Stoffe und als Ersatz des zerfallenen Gewebes. Ein Ersatz von Eiweiss durch Leim in der ersteren Funktion ist kaum zu bezweifeln. Nach J. Munk's Berechnung ${ }^{3}$ ) der Voit'schen Versuche ersparen im Mittel $100 \mathrm{~g}$ Leim $150 \mathrm{~g}$ Fleisch $=31 \mathrm{~g}$ Eiweiss und $100 \mathrm{~g}$ Leim $200 \mathrm{~g}$ Kohlehydrate. Dagegen werden durch $100 \mathrm{~g}$ Leim nur etwa $25 \mathrm{~g}$ Fett erspart.

Joseph Kirchmann $n^{4}$ hat in seinen Versuchen gefunden, dass bei einer Leimeinfuhr, die ungefähr $12 \%$ des Energiebedarfs deckt, der Eiweisszerfall von 100 auf 75 , also um $25 \%$, fiel. „Mit grösseren Leimgaben lassen sich dann nur mehr ganz geringe Veränderungen erzielen. Die höchste Verminderung des Eiweisszerfalls betrug $35 \%$, obwohl die dabei gegebene Leimmenge $62 \%$ des Energiebedarfs zu decken imstande war. Sicher ist, dass der Maximal-

1) Zeitschr. f. physiol. Chemie Bd. 5 S. 31.

2) Archiv für experimentelle Pathologie und Pharmakologie Bd. 48 S. 396.

3) Real-Enzyklopädie der ges. Heilkunde. Leimstoffe.

4) Zeitschrift für Biologie Bd. 40 S. 54.

E. Pflüger, Archiv für Physiologie. Bd. 109. 
wert der Leimwirkung oder vielmehr der geringste Eiweisszerfall nicht weit unter die Grösse 64,9 herabsinken kann." In allen seinen Versuchen wird durch die Leimzufuhr der Eiweisszerfall zwar vermindert, aber durchaus nicht völlig aufgehoben.

J. $M \mathrm{nk}^{1}$ ) hat seine Versuche mit Leim so eingerichtet, dass die Stickstoffeinnahme in Eiweiss- und Leimperiode gleichblieb. Als er $5 / 6$ des sehr reichlich gegebenen Eiweisses durch Leim mit gleichem Stickstoffgehalt ersetzte, wurde die positive Stickstoffbilanz nur um etwa $0,3 \mathrm{~g}$ ungünstiger.

In neuerer Zeit hat Karl Gregor ${ }^{2}$ ) die Bedeutung des Leims als Gewebe bildend in der Säuglingsnahrung hervorgehoben.

Auf Anregung von Professor $\mathrm{Zuntz}$ unternahm es Verfasser, durch Stoffwechselversuche bei Hunden nachzuprüfen, 1. wieviel Eiweiss durch Leim bei Stickstoffgleichgewicht ersetzt werden könne, und 2. durch Zusatz von nicht in Leim enthaltenen Spaltungsprodukten des Eiweisses zum Leim eine eventuelle Eiweisssynthese im Tierkörper zu versuchen.

Nach der Annahme von Zuntz muss der Leim, soweit es sich um den Ersatz des leimgebenden Gewebes im Körper handelt, nicht nur dem Eiweiss gleichwertig, sondern ihm sogar überlegen sein, wenn man bedenkt, dass, ehe der Aufbau des leimgebenden Gewebes durch das Eiweiss zustande kommt, von diesem letzteren einige Moleküloruppen abgespalten, vielleicht auch andere angelagert werden müssen. Wie gross diese Menge Leim im Verhältnis zum Gesantstickstoff des Körpers sei, kann man a priori nicht abseben. Wir kennen zwar annähernd das Mengenverhältnis des leimgebenden Gewebes in den Knochen, Sehnen usw. zum eigentlichen Eiweiss des Körpers, aber wir kennen nicht die Intensität des Zerfalls in diesem Gewebe. Wenn wir im Versuch die Grenze ermitteln können, bis zu der Leim vollwertig das Eiweiss als Nahrungsmittel vertreten kann, dürften die so gewonnenen Zablen wohl einen Anhaltspunkt für den Umfang geben, in welchem das leimgebende Gewebe zerfällt und durch Nahrungsmaterialien ersetzt werden muss. - Aufgabe der Untersuchung war also, zu ermitteln, wie weit das Eiweiss als Gewebsbildner durch Leim zu ersetzen ist. $\mathrm{Zu}$ diesem Behufe musste schon in der Eiweissperiode die ein-

1) Pflüger's Archiv Bd. 58 S. 309. Virchow's Archiv Bd. 101 p. 110.

2) Zentralblatt für innere Medizin Bd. 22 S. 65. 1901. 
geführte Eiweissmenge so gering, die N-freie Nahrung so reichlich bemessen werden, dass das Eiweiss als Brennstoff möglichst ausgeschaltet war; von diesem zur Erhaltung des N-Bestandes im Körper unentbehrlichen Eiweiss sollte dann ein Teil durch Leim ersetzt werden.

Durch die Versuche von Landergren ${ }^{1}$ ) ist festgestellt worden, dass, je geringer die $\mathrm{N}$-Einfuhr bei reichlichem Brennwert der Nahrung ist, desto geringer auch die N-Ausgabe. Bei Ernährung mit nahezu stickstofffreier Kost konnte Lan d e r g r en den N-Verlust auf ein Minimum herabdrücken. Diese Resultate sollten bei den Tierversuchen berücksichtigt werden.

I.

Abweichend von den früheren Versuchsanordnungen wurde das Fleisch von der Nahrung ausgeschlossen, da es ja wechselnde Mengen von leimgebendem Gewebe entbält, also keinen strengen Vergleich von leimhaltiger und leimfreier Nahrung gestattet.

Für die Versuche standen zwei gesunde Hündinnen zur Verfügung; die grössere war etwa $19 \mathrm{~kg}$ schwer, die kleinere ca. $9 \mathrm{~kg}$, so dass man der grösseren etwa doppelt so viel Nahrung geben konnte als der kleineren. Weibliche Tiere wurden bevorzugt, weil sie leichter zu katheterisieren waren. Die Hündinnen waren gesund; der Urin war frei von Eiweiss und Zucker, sauer reagierend.

Während des Versuchs wurden die Tiere je in einem (der im Laboratorium gebräuchlichen geräumigen) Käfige gehalten, dessen Deckel nur von einzelnen Eisenstäben gebildet wurde und hinreichend Licht und Luft zuliess. Der Boden bestand ebenfalls aus parallelen Eisenstäben, die nur $1 \mathrm{~cm}$ voneinander entfernt waren, so dass der etwa entleerte Urin in eine darunter befindliche Schublade lief, während der Kot auf den Eisenstäben liegen blieb. Diese Schublade war mit Zinkblech ausgeschlagen; an ihrer tiefsten Stelle lief ein Rohr nach aussen, unter welches ein Uringlas gestellt wurde.

Die Käfige wurden von mir und einem alten, erfahrenen Diener oftmals während des Tages inspiziert, etwaiger Kot gesammelt; nach etwaiger Urinentleerung wurde der Käfig mit Wasser nachgespült. Jeden Tag um dieselbe Zeit wurde katheterisiert, die Blase mit Borsäure ausgespült; danach wurden die Tiere einige Minuten ins

1) Skandinav. Archiv Bd. 14 S. 112. 
Freie geführt, wobei meist Defäkation stattfand; eventuell wurde dieselbe durch Einführung eines dicken, angefeuchteten Glasstabes in den After herbeigeführt. Beide Tiere gewöhnten sich schon nach einigen Tagen daran, Urin und Kot 24 Stunden zu halten. Die Nahrung wurde zu einem Brei zusammengekocht und nach dem Exkalten vorgesetzt.

Von sämtlichen Nahrungsmitteln wurde der N-Gehalt bestimmt. Die Stickstoffbestimmung geschah nach Kjeldahl. Es wurde mit Schwefelsäure unter Zugabe eines kleinen Tropfens Quecksilbers mindestens drei Stunden aufgeschlossen. Den Berechnungen der Kalorien wurden die aus den Tabellen von Landolt und Börnstein entnommenen Stohmann'schen Daten zugrunde gelegt; die Abgrenzung des Kotes geschah durch feingepulverte Kieselsäure; der Kot wurde von jeder Periode gesammelt, getrocknet und fein gemahlen.

Als Ersatz von Fleisch wurde Kasein in Form des Plasmons (Kasein-Na) gewählt, von dem $\mathrm{Caspari}{ }^{1}$ ) durch Versuche nachgewiesen hat, dass es besser resorbiert wird als Fleisch. Das Plasmon ist geruchlos, fast geschmacklos, leicht löslich. Sterilisierte Milch lieferte die Meierei von Bolle; die einzelnen Flaschen entstammten demselben Mischgefäss. Die Gelatine wurde von Schering bezogen. Sie verlor im Trockenschrank 15\% Wasser, war in Essigsäure leicht löslich und vollkommen eiweissfrei. Die essigsaure Lösung wurde weder durch Zusatz von Ferrocyankalium noch durch Zusatz von Salpetersäure getrübt; bei Kochen mit Millon'schem Reagens entstand keine Rotfärbung.

Es wurden ferner noch Schweinefett und beim ersten Versuch als weiteres stickstoffhaltiges Nahrungsmittel gemablener Reis gegeben. Da die Nabrung sonst genügend Salze enthielt, so wurde nur wegen der Eisenarmut der Milch etwas Ferrum lacticum zugesetzt, und zwar beim grossen Hund $0,1 \mathrm{~g}$, beim kleinen $0,05 \mathrm{~g}$.

Für den grossen Hund mit einem Anfangsgewicht von $19430 \mathrm{~g}$ wurden pro Kilogramm $62 \mathrm{Kal}$. und $0,31 \mathrm{~g} \mathrm{~N}$ angenommen. $\mathrm{Er}$ erhielt demnach :

$600 \mathrm{ecm}$ Milch mit $0,527 \% \mathrm{~N}$; pro Kubikzentimeter 0,648 Kal., $100 \mathrm{~g}$ Reismehl " $1,35 \% \mathrm{~N}$; "Gramm 4,1 "

$38 \mathrm{~g} \quad$ Fett

$12 \mathrm{~g}$ Plasmon "12,72 \% N; " " $\quad 4,1$ "

1) Fortschritte der Medizin 1899 Nr. 19. 
also:

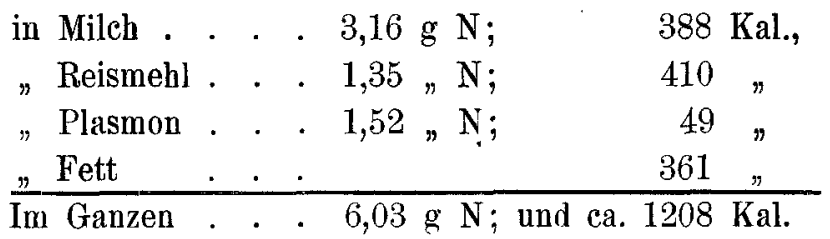

Da nach drei Tagen das Körpergewicht um $70 \mathrm{~g}$ gefallen war, so wurde die Plasmongabe von 12 auf $20 \mathrm{~g}$ erhöht, so dass dann im ganzen 7,05 g N und 1241 Kal., pro Kilogramm 64 Kal. gegeben wurden.

Vun diesen $7,05 \mathrm{~g} \mathrm{~N}$ sollte nun der fünfte Teil, $1,41 \mathrm{~g} \mathrm{~N}$, ersetzt werden durch Gelatine-N. Da die Gelatine $15,13 . \% \mathrm{~N}$ enthielt, so entsprachen 9,33 $\mathrm{g}$ Gelatine $1,41 \mathrm{~g} \mathrm{~N}$ oder $11,2 \mathrm{~g}$ Plasmon. Für Gelatine wurden pro Gramm 4,4 Kal. angenommen, so dass die Kalorieneinfuhr als gleich gelten kann.

Tabelle I. Grosser Hund.

Ersatz von $1 / 5$ Eiweiss-N durch Leim-N.

\begin{tabular}{|c|c|c|c|c|c|c|}
\hline Datum & $\begin{array}{c}\text { Gewicht } \\
\text { g }\end{array}$ & $\begin{array}{l}\text { N-Ein- } \\
\text { nahme }\end{array}$ & $\begin{array}{c}\mathrm{N}- \\
\text { Ausgabe } \\
\text { Urin }\end{array}$ & Kot-N & $\begin{array}{l}\text { Gesamt- } \\
\text { N- } \\
\text { Ausgabe }\end{array}$ & Bilanz \\
\hline $\begin{array}{l}\text { 11. Nov. } \\
\text { 12. " } \\
13 . "\end{array}$ & $\begin{array}{l}19430 \\
19400 \\
19360 \mathrm{Kot}\end{array}$ & $\begin{array}{l}6,03 \\
6,03 \\
6,03\end{array}$ & $\begin{array}{l}3,84 \\
4,73 \\
6,34\end{array}$ & $\begin{array}{l}0,39 \\
0,39 \\
0,39\end{array}$ & $\begin{array}{l}4,23 \\
5,12 \\
6,73\end{array}$ & $\begin{array}{l}+1,80 \\
+0,91 \\
+0,70\end{array}$ \\
\hline $\begin{array}{l}\text { 14. Nov. } \\
\text { 15. " } \\
16 . " \\
17 . " \\
18 . "\end{array}$ & $\mid \begin{array}{l}19270 \\
19260 \text { Kot } \\
19210 \\
19190 \text { Kot } \\
19160\end{array}$ & $\begin{array}{l}\text { Vo } \\
7,05 \\
7,05 \\
7,05 \\
7,05 \\
7,05\end{array}$ & $\begin{array}{c}\text { periode } \\
5,47 \\
5,93 \\
6,14 \\
5,76 \\
6,08\end{array}$ & $\begin{array}{l}0,39 \\
0,39 \\
0,39 \\
0,39 \\
0,39\end{array}$ & $\begin{array}{l}5,86 \\
6,32 \\
6,53 \\
6,15 \\
6,47\end{array}$ & $\begin{array}{l}+1,19 \\
+0,73 \\
+0,52 \\
+0,90 \\
+0,58\end{array}$ \\
\hline & & & Mittel & . . & 6,26 & $+0,78$ \\
\hline $\begin{array}{l}\text { 19. Nov. } \\
20 . " ~ \\
21 . " ~ \\
22 . \\
23 . " ~ \\
24 .\end{array}$ & $\begin{array}{l}19160 \mathrm{Kot} \\
19230 \\
19240 \mathrm{Kot} \\
19260 \\
19270 \mathrm{Kot} \\
19220\end{array}$ & $\begin{array}{c}\text { Lei } \\
7,05 \\
7,05 \\
7,05 \\
7,05 \\
7,05 \\
7,05\end{array}$ & $\begin{array}{c}\text { erio de } \\
5,98 \\
5,80 \\
5,98 \\
5,71 \\
5,66 \\
5,29 \\
\end{array}$ & $\begin{array}{l}0,39 \\
0,39 \\
0,39 \\
0,39 \\
0,39 \\
0,39 \\
\end{array}$ & $\begin{array}{l}6,37 \\
6,19 \\
6,37 \\
6,10 \\
6,05 \\
5,68 \\
\end{array}$ & $\begin{array}{l}+0,68 \\
+0,86 \\
+0,68 \\
+0,95 \\
+1,00 \\
+1,37\end{array}$ \\
\hline & & & Mittel & & 6,13 & $+0,92$ \\
\hline $\begin{array}{l}25 . \\
26 . \\
27 . " ~ \\
28 . \\
29 . \quad "\end{array}$ & $\begin{array}{l}19180 \text { Kot } \\
19220 \\
19180 \text { Kot } \\
19220 \\
19240 \text { Kot }\end{array}$ & $\begin{array}{l}\mathrm{Na} \\
7,05 \\
7,05 \\
7,05 \\
7,05 \\
7,05\end{array}$ & $\begin{array}{l}\text { e ri od } \\
5,83 \\
5,86 \\
5,95 \\
5,68 \\
5,60\end{array}$ & $\begin{array}{l}0,37 \\
0,37 \\
0,37 \\
0,37 \\
0,37\end{array}$ & $\begin{array}{l}6,20 \\
6,23 \\
6,82 \\
6,05 \\
5,97\end{array}$ & $\begin{array}{l}+0,85 \\
+0,82 \\
+0,73 \\
+1,00 \\
+1,08\end{array}$ \\
\hline & & & Mitte & & 6,13 & $+0,89$ \\
\hline
\end{tabular}


Die Tage vom 11. bis 13 . November wurden nicht mitgerechnet; die Bilanz ist fast in allen drei Perioden gleich, ein Fünftel Eiweiss-N wurde glatt durch Leim-N ersetzt. Der Ủberschuss an Stickstoff in allen drei Perioden beweist, dass die $\mathrm{N}$-Zahl, pro Kilogramm $0,37 \mathrm{~g}, \mathrm{zu}$ hoch angenommen wurde. Die Leimperiode zeigt sogar eine bessere Bilanz als die beiden anderen, was der Ansicht von $\mathrm{Zuntz}$ entsprechen würde. J. $\mathrm{Munk}^{1}$ ) hat sogar $5 / 6$ des Eiweiss-N durch Leim-N ersetzt, aber hier war die absolute Menge des Eiweisses sehr gross, und die Hauptmenge desselben diente einfach als Energiequelle.

Für den kleinen Hund nit einem Anfangsgewicht von $9350 \mathrm{~g}$ wurde in Anbetracht der verhältnismässig grösseren Körperoberfläche die Hälfte der Nahrung des grossen Hundes ausgerechnet, also:

\begin{tabular}{rlllll}
$300 \mathrm{ccm}$ & Milch &. &. &. & $1,581 \mathrm{~g} \mathrm{~N} ; 194$ Kal. \\
$50 \mathrm{~g}$ & Reis &. &. &. & $0,67 \mathrm{~g} \mathrm{~N} ; 205 " ~$ \\
$6 \mathrm{~g} \quad$ Plasmon &. &. & $0,763 \mathrm{~g} \mathrm{~N} ; 24 "$ \\
$19 \mathrm{~g}$ Fett &. &. & 180 \\
\hline Im ganzen . &. &. &. & $3,015 \mathrm{~g} \mathrm{~N} ; 603 \mathrm{Kal}$. \\
oder pro Kilogramm &. & $0,32 \mathrm{~g} \mathrm{~N} ; 64,5 \mathrm{Kal}$.
\end{tabular}

Wegen stärkerer Gewichtsabnahme wurden vom vierten Tage an $10 \mathrm{~g}$ Plasmon und $25 \mathrm{~g}$ Fett gegeben, also im ganzen $3,52 \mathrm{~g} \mathrm{~N}$ und pro Kilogramm 72 Kal.

Davon sollte der dritte Teil des Stickstoffs oder 1,17 g N durch Gelatine-N ersetzt werden. Es wurden also $7,73 \mathrm{~g}$ Gelatine statt 9,2 g Plasmon gegeben, wodurch die Zahl der eingeführten Kalorien nicht verändert wurde. Für die Berechnung wurden wieder die drei ersten Tage ausgeschaltet. Da der Hund am fünften Tage nur widerwillig frass, wurde statt des sechsten Gelatinetages gleich die Nachperiode angeschlossen.

Tabelle I. Kleiner Hund.

Ersatz von 1/3 Eiweiss-N durch Leim-N.

\begin{tabular}{|c|c|c|c|c|c|c|}
\hline Datum & $\begin{array}{c}\text { Gewicht } \\
\mathrm{g}\end{array}$ & $\begin{array}{c}\mathrm{N}- \\
\text { Einnahme }\end{array}$ & $\begin{array}{c}\mathrm{N}- \\
\text { Ausgabe } \\
\text { Urin }\end{array}$ & Kot-N & $\begin{array}{c}\text { Gesamt- } \\
\mathrm{N}- \\
\text { Ausgabe }\end{array}$ & Bilanz \\
\hline $\begin{array}{l}\text { 11. Nov. } \\
12 . " ~ \\
13 . "\end{array}$ & $\begin{array}{l}9350 \text { Kot } \\
9330 \text { Kot } \\
9270 \text { Kot }\end{array}$ & $\begin{array}{l}3,015 \\
3,015 \\
3,015\end{array}$ & $\begin{array}{l}2,31 \\
2,39 \\
2,59\end{array}$ & $\begin{array}{l}0,53 \\
0,58 \\
0,53\end{array}$ & $\begin{array}{l}2,84 \\
2,92 \\
3,12\end{array}$ & $\begin{array}{r}+0,17 \\
+0,09 \\
-0,01\end{array}$ \\
\hline
\end{tabular}

1) Pflüger's Archiv Bd. 58 S. 309. 


\begin{tabular}{|c|c|c|c|c|c|c|}
\hline Datum & $\begin{array}{c}\text { Gewicht } \\
\mathrm{g}\end{array}$ & $\begin{array}{c}\mathrm{N}- \\
\text { Einnahme }\end{array}$ & $\begin{array}{c}\text { N- } \\
\text { Ausgabe } \\
\text { Urin }\end{array}$ & Kot-N & $\begin{array}{l}\text { Gesamt- } \\
\mathrm{N}- \\
\text { Ausgabe }\end{array}$ & $\operatorname{Bilan} z$ \\
\hline \multicolumn{7}{|c|}{ Vorperiode: } \\
\hline \multirow[t]{2}{*}{$\begin{array}{l}\text { 14. Nov. } \\
15 . " ~ \\
16 . " ~ \\
17 . " \\
18 . "\end{array}$} & $\begin{array}{l}9220 \text { Kot } \\
9200 " \\
9170 " \\
9130 " \\
9100 "\end{array}$ & $\begin{array}{l}3,52 \\
3,52 \\
3,52 \\
3,52 \\
3,52\end{array}$ & $\begin{array}{l}2,84 \\
2,81 \\
2,92 \\
3,00 \\
3,09 \\
\end{array}$ & $\begin{array}{l}0,53 \\
0,53 \\
0,53 \\
0,53 \\
0,53 \\
\end{array}$ & $\begin{array}{l}3,37 \\
3,34 \\
3,45 \\
3,53 \\
3,62 \\
\end{array}$ & $\begin{array}{l}+0,15 \\
+0,18 \\
+0,07 \\
-0,01 \\
-0,1\end{array}$ \\
\hline & & & \multicolumn{2}{|c|}{ Mittel . } & 3,46 & $+0,06$ \\
\hline \multirow[t]{2}{*}{$\begin{array}{l}\text { 19. Nov. } \\
20 . " ~ " \\
21 . \\
22 . " ~ " ~ \\
23 . \quad "\end{array}$} & $\begin{array}{l}9050 \text { Kot } \\
8950 " n \\
8980 " \\
8850 " \\
8880 "\end{array}$ & $\begin{array}{l}\text { L e i m } \\
3,52 \\
3,52 \\
3,52 \\
3,52 \\
3,52\end{array}$ & $\begin{array}{c}\text { eri o d e: } \\
4,35 \\
3,60 \\
3,03 \\
4,09 \\
3,77 \\
\end{array}$ & $\begin{array}{l}0,62 \\
0,62 \\
0,62 \\
0,62 \\
0,62 \\
\end{array}$ & $\begin{array}{l}4,97 \\
4,22 \\
3,65 \\
4,71 \\
4,39 \\
\end{array}$ & $\begin{array}{l}-1,45 \\
-0,70 \\
-0,13 \\
-1,19 \\
-0,87 \\
\end{array}$ \\
\hline & & & \multicolumn{2}{|c|}{ Mittel . } & $\mathbf{4 , 3 9}$ & $-0,87$ \\
\hline \multicolumn{7}{|c|}{ Nachperiode: } \\
\hline \multirow[t]{2}{*}{ 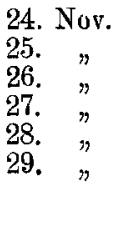 } & $\begin{array}{l}8880 \text { Kot } \\
8780 \text { " } \\
8680 \text { " } \\
8690 \text { " } \\
8670 \text { " } \\
8620 \text { " }\end{array}$ & $\begin{array}{l}3,52 \\
3,52 \\
3,52 \\
3,52 \\
3,52 \\
3,52\end{array}$ & $\begin{array}{l}2,77 \\
2,97 \\
2,52 \\
2,64 \\
2,28 \\
\mathbf{2 , 4 3} \\
\end{array}$ & $\begin{array}{l}0,58 \\
0,58 \\
0,58 \\
0,58 \\
0,58 \\
0,58\end{array}$ & $\begin{array}{l}3,35 \\
3,55 \\
3,10 \\
3,22 \\
2,86 \\
3,01 \\
\end{array}$ & $\begin{array}{l}+0,17 \\
+0,03 \\
+0,42 \\
+0,30 \\
+0,66 \\
+0,51\end{array}$ \\
\hline & & & \multicolumn{2}{|c|}{ Mittel . . } & 3,36 & $+0,37$ \\
\hline
\end{tabular}

Die Bilanz zeigt eine beträchtliche Vermehrung der Stickstoffausgabe in der Hauptperiode, ferner eine Vermehrung des Kot-N in der zweiten und dritten Periode. Das Eiweiss wurde also durch den Leim nicht ersetzt; vielmehr stieg in der Leimperiode der $\mathrm{N}$-Zerfall beinahe um den Wert des zugeführten Leimes, denn der $\mathrm{N}$-Ansatz betrug:

$$
\begin{aligned}
& \text { im Mittel der Vor- und Nachperiode }+0,21 \mathrm{~g}, \\
& \text { in der Leimperiode }
\end{aligned}
$$

also eine Schädigung der Stickstoffbilanz um 1,08 $\mathrm{g}$ bei einer Gesamtmenge von $1,17 \mathrm{~g}$ Leim-N.

Die Tiere wurden nun wieder mit Fleischkost gefüttert; am 4. Dezember begann der nächste Versuch mit einem Hungertag, um das Stickstoffgleichgewicht rascher herbeizuführen und die Tiere fresslustiger zu machen. Es bekam der grosse Hund am 4. Dezember $300 \mathrm{ccm}$ Milch und $50 \mathrm{~g}$ Stärke, der kleine Hund $150 \mathrm{ccm}$ Milch und $25 \mathrm{~g}$ Stärke.

Am 5. Dezember wurde der zweite Versuch begonnen. Der grosse Hund bekam: 
$300 \mathrm{ccm}$ Milch mit $0,538 \% \mathrm{~N}$; pro Kubikzentimeter $0,648 \mathrm{Kal}$. 30 g Plasmon mit 12,72 \% N; pro Gramm 4,1 ” $120 \mathrm{~g}$ Stärke mit $0,42 \% \mathrm{~N}$; pro " 4,1 " $50 \mathrm{~g}$ Fett $0,1 \mathrm{~g}$ Ferrum lacticum pro also:

$$
\begin{aligned}
\text { in Milch } & =1,616 \mathrm{~g} \mathrm{~N} ; 194 \text { Kal. } \\
\text { "Plasmon } & =3,816 \mathrm{~g} \mathrm{~N} ; 123 " \\
\text { "Stärke } & =0,05 \mathrm{~g} \mathrm{~N} ; 492 " \\
\text { "Fett } & = \\
\text { Im ganzen } & =5,48 \mathrm{~g} \mathrm{~N} ; 1284 \text { Kal. } \\
\text { oder pro Kilogramm } & =0,3 \mathrm{~g} \mathrm{~N} ; 69 "
\end{aligned}
$$

Es wurde also die N-Einfuhr vermindert und in Anbetracht der Körpergewichtsabnahme im ersten Versuch die Kalorieneinfubr erhöht. Von den $5,48 \mathrm{~g} \mathrm{~N}$ sollte wieder ein Fünftel, also $1,9 \mathrm{~g}$ durch

\begin{tabular}{|c|c|c|c|c|c|c|}
\hline Datum & $\begin{array}{c}\text { Gewicht } \\
\mathrm{g}\end{array}$ & $\begin{array}{c}\mathrm{N}- \\
\text { Einnahme }\end{array}$ & $\begin{array}{c}\text { N- } \\
\text { Ausgabe } \\
\text { Urin }\end{array}$ & Kot-N & $\begin{array}{c}\text { Gesamt- } \\
\mathrm{N}- \\
\text { Ausgabe }\end{array}$ & Bilanz \\
\hline $\begin{array}{l}\text { 4. Dez. } \\
5 . " \Rightarrow \\
6 . " \Rightarrow\end{array}$ & $\begin{array}{l}18500 \mathrm{Kot} \\
18400 \mathrm{Kot} \\
18300 \mathrm{Kot}\end{array}$ & $\begin{array}{l}1,64 \\
5,48 \\
5,48\end{array}$ & $\begin{array}{l}2,88 \\
3,99 \\
3,13\end{array}$ & $\begin{array}{c}? \\
0,5 \\
0,5\end{array}$ & $\begin{array}{l}\overline{4,49} \\
3,63\end{array}$ & $\begin{array}{c}-(1,24+\mathrm{x}) \\
+0,99 \\
+1,85\end{array}$ \\
\hline
\end{tabular}
Gelatine-N ersetzt werden; somit musste statt $8,7 \mathrm{~g}$ Plasmon $7,24 \mathrm{~g}$ Gelatine gegeben werden, wobei die Kalorienzahl dieselbe blieb.

Tabelle II. Grosser Hund.

Ersatz von $1 / 5$ Eiweiss-N durch Leim-N.

Vorperiode.

\begin{tabular}{rl|l|l|l|l|l|l} 
7. & Dez. & 18400 & 5,48 & 4,40 & 0,5 & 4,90 & $+0,58$ \\
8. & 18370 Kot & 5,48 & 4,37 & 0,5 & 4,87 & $+0,61$ \\
9. & $18350 \%$ & 5,48 & 4,23 & 0,5 & 4,73 & $+0,75$ \\
10. & $"$ & $18450 \%$ & 5,48 & 4,53 & 0,5 & 5,03 & $+0,45$ \\
\hline
\end{tabular}

Leimperiode.

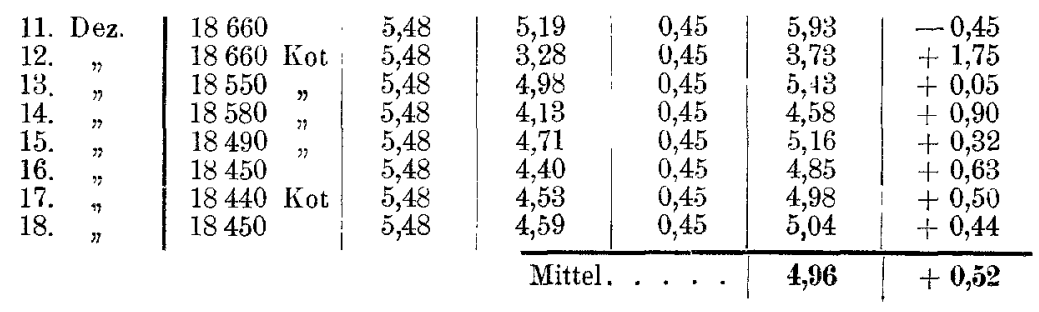




\begin{tabular}{|c|c|c|c|c|c|c|}
\hline Datum & $\begin{array}{l}\text { Gewicht } \\
\mathrm{g}\end{array}$ & $\begin{array}{c}\mathrm{N}- \\
\text { Einnahme }\end{array}$ & $\begin{array}{c}\mathrm{N}- \\
\text { Ausgabe } \\
\text { Urin }\end{array}$ & Kot-N & $\begin{array}{c}\text { Gesamt- } \\
\mathrm{N} \text { - } \\
\text { Ausgabe }\end{array}$ & Bilanz \\
\hline \multicolumn{7}{|c|}{ Nachperiode. } \\
\hline \multirow{2}{*}{ 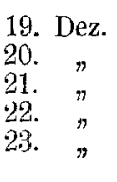 } & \multirow{2}{*}{$\begin{array}{l}18390 \text { Kot } \\
18410 " \\
18300 " \\
18200 " \\
18150 "\end{array}$} & $\begin{array}{l}5,48 \\
5,48 \\
5,48 \\
5,48\end{array}$ & $\begin{array}{l}5,29 \\
4,69 \\
4,87 \\
4,41\end{array}$ & $\begin{array}{l}0,50 \\
0,50 \\
0,50 \\
0,50\end{array}$ & $\begin{array}{l}5,79 \\
5,19 \\
4,87 \\
4,91\end{array}$ & $\begin{array}{r}-0,31 \\
+0,29 \\
+0,61 \\
+0,57\end{array}$ \\
\hline & & & Mitte & & 5,19 & $+0,29$ \\
\hline
\end{tabular}

Der erste Tag konnte nicht mitgerechnet werden, weil er noch unter der Wirkung des vorhergehenden Futters steht; auch am 6. Dezember hatte sich der Körper noch nicht auf die N-Einfuhr eingestellt; die Bilanz wurde erst vom 7. Dezember an begonnen. Sie zeigt ein Fortschreiten der N-Ausgabe zu ungunsten der dritten Periode; auffallend ist die geringere N-Zahl für Kot in der zweiten Periode.

Nimmt man wieder das Mittel aus der ersten und dritten Periode, so beträgt die N-Bilanz

$$
\begin{aligned}
& \text { in der Eiweissperiode. } .+.+0,45 \mathrm{~g} \mathrm{~N} \text {, } \\
& \text { in der Leimperiode . . . . + }+0,52 \mathrm{~g} \mathrm{~N} \text {, }
\end{aligned}
$$

d. h. wieder ein geringer, aber noch innerhalb der Fehlergrenzen liegender Mehransatz in der Leimperiode.

Der kleine Hund bekam vom 5. Dezember ab

$$
\begin{aligned}
& 300 \mathrm{cem} \text { Milch . . . 1,616 g N; } 194 \text { Kal. } \\
& 10 \mathrm{~g} \text { Plasmon . . . } 1,272 \mathrm{~g} \mathrm{~N} ; 41 \mathrm{Kal} \text {. } \\
& 60 \text { g Stärke . . . . } 0,02 \text { g N; } 246 \text { Kal. } \\
& 30 \mathrm{~g} \text { Fett . . . } 285 \text { Kal. } \\
& 0,05 \mathrm{~g} \text { Ferrum lacticum } \\
& \text { Im ganzen . . . . . . } 2,91 \mathrm{~g} \mathrm{~N} ; 766 \text { Kal. }
\end{aligned}
$$

Auf das Gewicht vom 5. Dezember $=8110 \mathrm{~g}$ berechnet, sind das pro Kilogramm 94 Kal. und fast $0,36 \mathrm{~g}$ N. Beide Zahlen wurden so hoch postuliert, weil im ersten Versuch die Gewichtsabnahme eine ganz erhebliche gewesen war.

Von den 2,91 g $\mathrm{N}$ sollte der vierte Teil $0,763 \mathrm{~g} \mathrm{~N}$ durch Gelatine-N ersetzt werden, also statt $6 \mathrm{~g}$ Plasmon 5,04 g Gelatine. Die Kalorienzahl blieb wieder gleich. In der zweiten Periode wurde der Hund, um eine lange Reihe zu erhalten, so lange mit Leim gefüttert, als er die Nahrung willig nahm: neun Tage. 
Tabelle II. Kleiner Hund.

Ersatz von $1 / 4$ Eiweiss-N durch Leim-N.

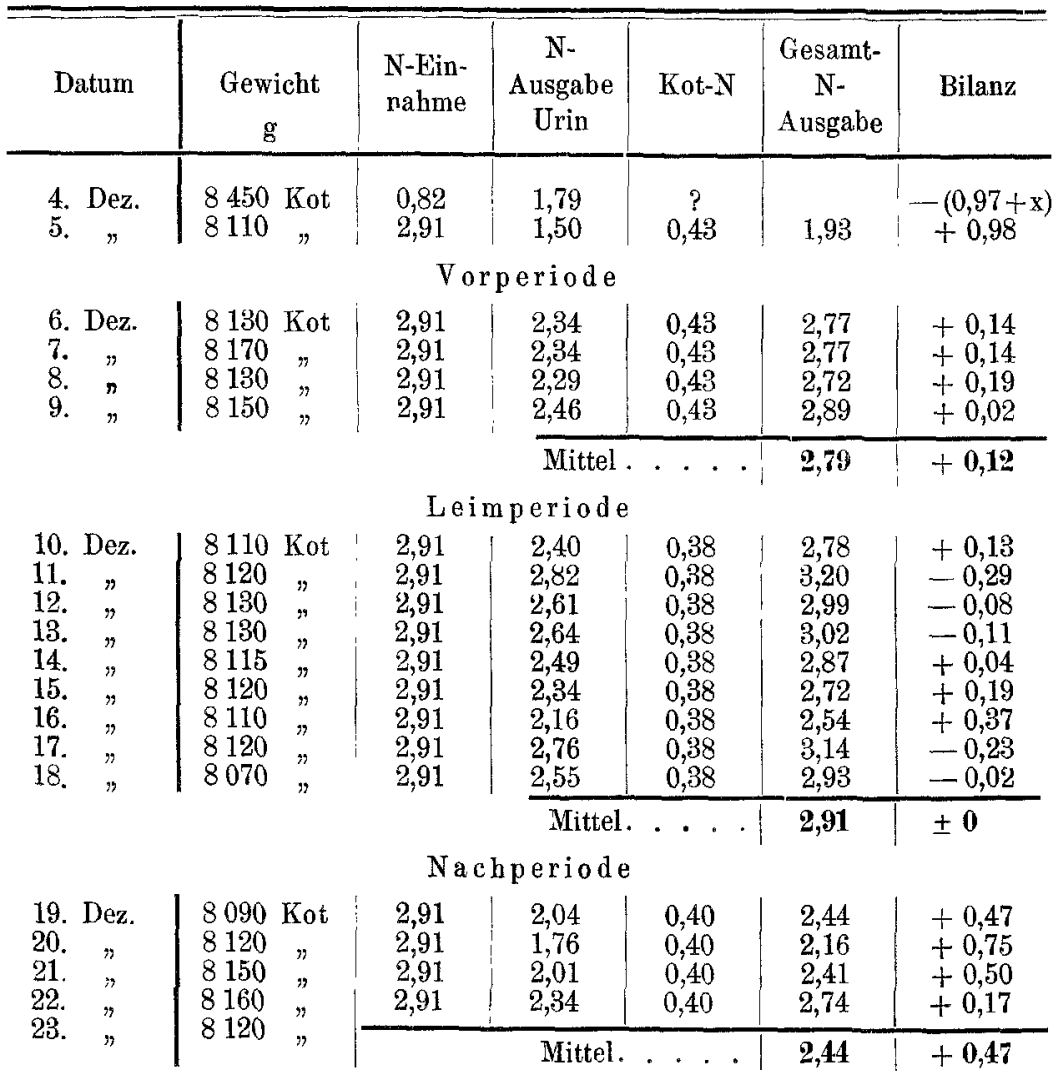

Die Bilanz ergibt eine Vermehrung der N-Ausgabe in der zweiten Periode; im Mittel entspricht sie zwar der N-Einnahme, aber im Vergleich zur Eiweissperiode ist sie erhöht.

Auch hier ist die geringere $\mathrm{N}$-Ausgabe durch den Kot in der Gelatineperiode beachtenswert, was für die leichtere Resorbierbarkeit des Leims, die Senator ${ }^{1}$ ) und Uffelmann ${ }^{2}$ ) schon betont haben, spricht.

Die vier Versuche beweisen unter möglichstem Ausschluss von Fehlerquellen

1. dass in der Nahrung von Hunden, welche nur soviel Eiweiss erhalten, als bei genügender Auf-

1) Der fieberhafte Prozess S. 174. 1873.

2) Die Diät in akut-fieberhaften Krankheiten S. 81. 1877. 
nahme von Brennmaterial zur Erhaltung des Körperbestands nötig ist, einem Fünftel Eiweis-N dieselbe Menge Leim-N physiologisch gleichwertig, wahrscheinlich sogar überlegen ist. Nach den anfangs gemachten Ausführungen würde also von unserem täglichen Zerfall von Körpersubstanz ein Fünftel auf das leimgebende Gewebe bezogen werden müssen;

2. dass ein Fünftel auch die Grenze des vollwertigen Ersatzes von Eiweiss durch Leim ist, denn schon bei Ersatz von einem Viertel Eiweiss-N durch Leim-N tritt eine kleine Unterbilanz zuungunsten des Leims auf.

So wird durch die Tatsachen die bis dabin hypothetische Annahme von $J . \mathrm{Munk}^{1}$ ) bestätigt, dass, „wenn, wie in der Kost des Menschen, allerböchstens ein Fünftel der N-baltigen Substanzen sich in Form von Leim und vier Fünftel in Form von Eiweiss finden, der Leim als dem Eiweiss gleichwertig zu erachten ist".

II.

Im Anschluss an die Entdeckung von Otto Cobnheim²), dass im Verdanungsapparat das Eiweiss in viel grösserem Umfang, als man bis dahin angenommen hatte, zu kristallinischen Abbauprodukten zerlegt wird, hat Otto $\mathrm{Loewj}^{3}$ ) als erster mit Erfolg versucht, Abbauprodukte des Eiweisses zu verfüttern; es gelang ihm, bei einer Hündin durch Ernährung mit Pankreasverdauungsprodukten Stickstoffgleichgewicht zu erzielen; es glückte ihm also eine physiologische Eiweisssynthese, und zwar in einer elftägigen Versuchsreihe.

Da bereits geringe Mengen von Verdauungslösungen bei direkter Injektion in die Blutbahn äusserst giftig sind, so nimmt Loew ${ }^{4}$ ) an, dass bei der Eiweissverdaung eine tiefgehende Spaltung des Eiweissmoleküls im Darm stattfindet, dass die Spaltungsprodukte durch Bindekörper des Blutes aufgenommen werden, die je nach Bedarf der Organe diesen von ihrer Ladung abgehen. Als Resultat seines gelungenen Versuches kommt er zu dem Schluss, „dass die Summe der biuretfreien Endprodukte Nahrungseiweiss ersetzen, d. b. für alle Teile des im Stoffwandel zugrunde gehenden Körpereiweisses

1) Real-Enzyklopädie der ges. Heilkunde. Leimstoffe S. 405.

2) Chemie der Eiweisskörper.

3) Über Eiweisssynthese im Tierkörper S. 315.

4) Eiweisssynthese S. 324-325. 
eintreten kann. Also auch das Tier kann Eiweiss aufbauen und ist nicht auf Nahrungseiweiss angewiesen, sofern wir in der biuretgebenden Gruppe dessen Charakteristikum erblicken wollen".

Ebenso haben in neuerer Zeit $\mathrm{Abderhalden}$ und Peter Rona ${ }^{1}$ ) gezeigt, dass es gelingt, Mäuse mit einem durch Pankreatinverdauung aus Kasein gewonnenen, biuretfreien, zum grössten Teil aus Aminosäuren bestehenden Produkte ebensolange am Leben zu erhalten wie mit unverändertem Kasein selbst. „Der tierische Organismus vermag aus Aminosäuren und komplizierten, biuretfreien Produkten seinen Bedarf an Eiweiss vollkommen zu decken."

Der Leim ist nun sicher dem Eiweiss sehr nahestehend, denn er liefert eine grosse Anzahl von Abbauprodukten, welche mit denen des Eiweisses identisch sind. Es wurde daher die alte Idee von Hermann, die Escher zuerst ausgefüht hat, von mir wiederaufgenommen, durch Zusatz von Spaltungsprodukten des Eiweisses, die im Leim nicht oder nur in geringen Mengen gefunden worden sind, im Tierkörper eine Eiweisssynthese zu versuchen.

Es ist seit langem bekannt, dass der aromatische Atomkomplex im Leim fast vollständig fehlt, wenn auch $\mathrm{Maly}^{2}$ ) durch Oxydation des Leims eine Säure erhalten hat, die bei Behandlung mit Chromsäure Benzoesäure liefert; deun es handelt sich bei letzterer um ganz geringe Mengen. Nach J. M u nk erhält Leim $50 \% \mathrm{C}, 17,9 \% \mathrm{~N}$ und nur $0,6 \% \mathrm{~S}$, während Eiweiss $53,6 \% \mathrm{C}, 1$ bis $1,2 \% \mathrm{~S}$ und nur $16 \% \mathrm{~N}$ enthält.

Seit E. Fischer ${ }^{3}$ ) eine verbesserte Methode der Hydrolyse mit Salzsäure angegeben hat, sind die Spaltungsprodukte der verschiedenen Eiweissarten und des Leims genauer bekannt geworden. Aus den bisher veröffentlichten Ergebnissen älterer und neuerer Autoren habe ich mir die umstehende Tabelle zusammengestellt ${ }^{4}$ ):

1) Zeitschrift für physiol. Chemie Bd. 44 S. 198.

2) Monatshefte für Chemie Bd. $10 \mathrm{~S} .26$.

ஓ) Zeitschr. f. physiol. Chemie Bd. 33 S. 151. „Hydrolyse des Kaseins durch Salzsäure".

4) Zeitschr. f. physiol. Chemie Bd. 35 S. 70. Fischer, Levene und Aders, Über die Hydrolyse des Leims. Ebenda Bd. $36 \mathrm{~S}$. 268. Fischer und Abderh ald en, Hydrolyse des Oxyhämoglobins durch Salzsäure. Ebenda Bd. 36 S. 462. Fischer und Dörpinghaus, Hydrolyse des Horns. Taylor, Über Eiweissspaltung durch Bakterien. - Berichte der deutschen chemischen Gesellschaft Bd. 32 S. 2451, Bd. 33 S. 2370 , Bd. 33 S. 2383 . E. Fischer, l. c. Bd. 34 S. 3214. Kossel, Über den gegenwärtigen Stand der Eiweisschemie. 


\begin{tabular}{|c|c|c|c|c|c|c|c|}
\hline & $\begin{array}{l}\text { Leim } \\
\% \%\end{array}$ & Kasein & $\stackrel{\Xi}{\Xi}$ & 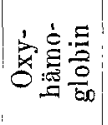 & 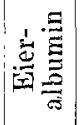 & 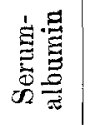 & 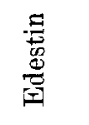 \\
\hline 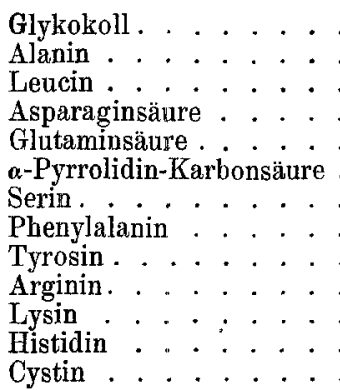 & $\begin{array}{c}16,5 \\
0,8 \\
\text { ca. } 4,0 \\
0,56 \\
0,88 \\
5,2 \\
? \\
0,4 \\
+ \\
+ \\
+ \\
+ \\
+\end{array}$ & $\begin{array}{c}\text { wahrseheinlich } \\
+ \\
\text { ca. } 5,0 \\
+ \\
+ \\
+ \\
+ \\
2,50 \\
>3,5 \text { (Colni) } \\
+ \\
+ \\
+ \\
+\end{array}$ & $\begin{array}{l}0,34 \\
1,20 \\
18,3 \\
2,5 \\
3,00 \\
3,6 \\
0,68 \\
3,0 \\
+ \\
+ \\
+ \\
+ \\
+\end{array}$ & $\begin{array}{r}4,02 \\
27,82 \\
4,25 \\
1,66 \\
2,25 \\
0,54 \\
3,38 \\
1,28 \\
1,0 \\
4,1 \\
10,5 \\
0,3\end{array}$ & $\begin{array}{l}+ \\
+ \\
+\end{array}$ & $\begin{array}{c}2,68 \\
20,0 \\
3,12 \\
1,52 \\
1,04 \\
0,6 \\
3,08 \\
2,1\end{array}$ & $\begin{array}{r}3,8 \\
3,6 \\
20,9 \\
4,5 \\
6,3 \\
1,7 \\
0,83 \\
1,4 \\
2,13 \\
2,0 \\
+ \\
1,1 \\
0,25\end{array}$ \\
\hline
\end{tabular}

Das Tyrosin fehlt im Leim; es fällt auf, wie gering die Menge der aromatischen Abbauprodukte im Leim ist, aber eine genaue prozentualisehe Berechnung der einzelnen Spaltungsprodukte im Leim einerseits, anderseits im Eiweiss liess sich aus den bisher erschienenen Arbeiten nicht entnehmen. Es ist nicht nur wichtig, zu wissen, welche Abbauprodukte als Zusatz zum Leim in Betracht kommen, sondern auch, wieviel Prozent des Leim-N durch Stickstoff von Eiweissabbauprodukten zu ersetzen ist. Denn es ist leicht möglich, dass beim Stoffwechselversuch ein Zuviel ebensowohl wie ein Zuwenig eines Spaltungsproduktes die physiologische Eiweisssynthese beeinträchtigen könnte.

Indol und Skatol wurden weder bei Fäulnis noch bei Hydrolyse mit Salzsäure aus Leim erbalten, während sie bekanntlich aus Eiweiss abspaltbar sind. Nun wurde von Hopkins und $\mathrm{Cole}^{1}$ ) eine Säure $\mathrm{C}_{11} \mathrm{H}_{12} \mathrm{~N}_{2} \mathrm{O}_{2}$ (Indolaminopropionsäure oder Skatolaminoessigsäure?) unter den tryptischen und Säurespaltungsprodukten von Fiweisskörpern (Kasein, Eieralbumin, Serumeiweiss) aufgefunden, die zur Fütterung geeignet erscheinen konnte.

Das Verhältnis von Monamino- und Diaminosäure im Leim sowohl wie im Eiweiss, womit sich $\mathrm{Hausmann}{ }^{2}$ ) beschäftigt hat, ist zurzeit noch unsicher.

Nach den Erfahrungen und Berechnungen von Dr. Abder-

1) Journal of Physiol. vol. 27 p. 418.

2) Zeitschrift für physiologische Chemie Bd. 27 S. 95. „Über die Verteilung des Stickstoffs im Eiweissmolekül." 
halden, der mich überhaupt in dankenswerter Weise beraten hat, empfahl es sich, $4 \%$ des Leim-N durch Tyrosin- $\mathrm{N}$ and $21 / 2 \%$ Leim-N durch Tryptophan-N zu ersetzen.

Das Tyrosin wurde durch tryptische Verdauung von Plasmon mit Pankreatin gewonnen. Selbsthergestelltes Tryptophan stellte mir erst Herr Dr. Oppenheimer, Assistent des Instituts, in liebenswürdiger Weise zur Verfügung; später habe ich es in grösseren Mengen aus Plasmon nach der von $\mathrm{Hopkins}$ und $\mathrm{Col}$ e angegebenen Methode hergestellt.

Es wurden wieder die beiden Hündinnen benützt.

Der grosse Hund sollte bekommen:

\begin{tabular}{|c|c|c|c|c|}
\hline $561 \mathrm{c}$ & cem Milch & $3,00 \quad \mathrm{~g} \mathrm{~N}$ & 365 & Kal. \\
\hline 11,9 & g Plasmon & $1,50 \mathrm{~g} \mathrm{~N}$; & 49 & \\
\hline 20 & Stärke & $0,001 \mathrm{~g} \mathrm{~N}$; & 82 & \\
\hline 58 & Zucker. & (3,9 Kal. pro g) & 226 & $"$ \\
\hline 50 & Fett. . & $. \quad . \quad \cdot \quad \cdot$. & 475 & \\
\hline
\end{tabular}

oder pro Kilogramm, da das Gewicht $17370 \mathrm{~g}$ betrug, $0,26 \mathrm{~g} \mathrm{~N}$ und fast $69 \mathrm{Kal}$.

Die N-Zahl wurde niedriger genommen als früher, um den in den früheren Versuchen beobachteten $\mathrm{N}$-Übersehuss zu vermeiden.

Von den $4,5 \mathrm{~g} \mathrm{~N}$ sollten $1,5 \mathrm{~g}$, also ein Drittel, durch Leim, kombiniert mit Tyrosin und Tryptophan, ersetzt werden.

Tyrosin p-Oxyphenylalanin $\mathrm{HO}(4) \mathrm{C}_{6} \mathrm{H}_{4} \mathrm{CH}_{2} \mathrm{CHNH}_{2} \mathrm{COOH}$ enthält $7,735 \% \mathrm{~N}$ und $5,9 \mathrm{Kal}$; davon mussten also zugegeben werden $0,776 \mathrm{~g}$.

Tryptophan $13,72 \% \mathrm{~N}$; Kal. ?, 0,273 g zu geben.

Ferner $9,26 \mathrm{~g}$ Gelatine.

Die übrige Nahrung blieb dieselbe, auch die Kalorienzahl blieb gleich. $\mathrm{Zu}$ bemerken ist noch, dass, während die Urinmenge in der ersten und dritten Periode täglich ca. $250 \mathrm{ccm}$ betrug, sie während der Gelatineperiode ca. $400 \mathrm{ccm}$ ausmachte.

Tabelle III. Grosser Hund.

1/3 Leim-N, 4\% Tyrosin, 2\% $1 \%$ Tryptophan.

\begin{tabular}{|c|c|c|c|c|c|c|}
\hline Datum & $\begin{array}{c}\text { Gewicht } \\
\mathrm{g}\end{array}$ & $\begin{array}{l}\text { N-Ein- } \\
\text { nahme }\end{array}$ & $\begin{array}{c}\text { N- } \\
\text { Ausgabe } \\
\text { Urin }\end{array}$ & Kot-N & $\begin{array}{c}\text { Gesamt- } \\
\text { N- } \\
\text { Ausgabe }\end{array}$ & Bilanz \\
\hline $\begin{array}{l}\text { 16. Jan. } \\
17 . " ~ \\
18 . "\end{array}$ & $\begin{array}{l}17370 \\
17420 \mathrm{Kot} \\
17440\end{array}$ & $\begin{array}{l}4,50 \\
4,50 \\
4,50\end{array}$ & $\begin{array}{l}4,86 \\
4,31 \\
4,25\end{array}$ & $\begin{array}{l}0,60 \\
0,60 \\
0,60\end{array}$ & $\begin{array}{l}5,46 \\
4,91 \\
4.85\end{array}$ & $\begin{array}{l}-0,96 \\
-0,41 \\
-0,35\end{array}$ \\
\hline
\end{tabular}


Über den Ersatz von Eiweiss durch Leim im Stoffwechsel.

\begin{tabular}{|c|c|c|c|c|c|c|}
\hline Datum & $\begin{array}{c}\text { Gewicht } \\
\mathrm{g}\end{array}$ & $\begin{array}{l}\text { N-Ein- } \\
\text { nahme }\end{array}$ & $\begin{array}{c}\mathrm{N}- \\
\text { A usgabe } \\
\text { Urin }\end{array}$ & Kot-N & $\begin{array}{c}\text { Gesamt- } \\
\mathrm{N}- \\
\text { Ausgabe }\end{array}$ & Bilanz \\
\hline \multicolumn{7}{|c|}{ Vorperiode } \\
\hline 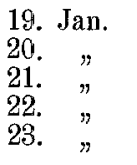 & $\begin{array}{l}17270 \\
17210 \text { Kot } \\
17200 \\
17150 \text { Kot } \\
17100\end{array}$ & $\begin{array}{l}4,50 \\
4,50 \\
4,50 \\
4,50 \\
4,50\end{array}$ & $\begin{array}{l}4,40 \\
3,76 \\
4,009 \\
4,11 \\
4,04\end{array}$ & $\begin{array}{l}0,60 \\
0,60 \\
0,60 \\
0,60 \\
0,60\end{array}$ & $\begin{array}{l}5,00 \\
4,36 \\
4,60 \\
4,71 \\
4,64\end{array}$ & $\begin{array}{l}-0,50 \\
+0,14 \\
-0,10 \\
-0,21 \\
-0,14\end{array}$ \\
\hline \multicolumn{6}{|c|}{$\begin{array}{l}\text { Mittel. } \\
\text { periode }\end{array}$} & $-0,16$ \\
\hline 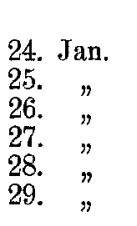 & $\begin{array}{l}17100 \\
17100 \\
17050 \mathrm{Kot} \\
17000 \\
16950 \mathrm{Kot} \\
16900 \mathrm{~L}\end{array}$ & $\begin{array}{l}\text { Lei } \\
4,50 \\
4,50 \\
4,50 \\
4,50 \\
4,50 \\
4,50\end{array}$ & $\begin{array}{c}\text { periode } \\
4,22 \\
4,32 \\
3,86 \\
4,16 \\
3,90 \\
4,14 \\
\end{array}$ & $\begin{array}{l}0,50 \\
0,50 \\
0,50 \\
0,50 \\
0,50 \\
0,50 \\
\end{array}$ & $\begin{array}{l}4,72 \\
4,82 \\
4,36 \\
4,66 \\
4,40 \\
4,64 \\
\end{array}$ & $\begin{array}{r}-0,22 \\
-0,32 \\
+0,14 \\
-0,16 \\
+0,10 \\
-0,14 \\
\end{array}$ \\
\hline \multicolumn{5}{|c|}{ Mittel. . . · } & 4,60 & $-0,10$ \\
\hline \multicolumn{7}{|c|}{ Nachperiode. } \\
\hline $\begin{array}{l}\text { 30. Jan. } \\
\text { 31. " " } \\
\text { 1. Febr. } \\
\text { 2. " " } \\
\text { 3. " }\end{array}$ & $\begin{array}{l}16900 \\
16850 \\
16800 \text { Kot } \\
16700 \\
16750\end{array}$ & $\begin{array}{l}4,50 \\
4,50 \\
4,50 \\
4,50 \\
4,50\end{array}$ & $\begin{array}{l}4,02 \\
4,10 \\
3,90 \\
4,02 \\
4,04\end{array}$ & $\begin{array}{l}0,71 \\
0,71 \\
0,71 \\
0,71 \\
0,71\end{array}$ & $\begin{array}{l}4,73 \\
4,81 \\
4,61 \\
4,73 \\
4,75\end{array}$ & $\begin{array}{l}-0,23 \\
-0,31 \\
-0,11 \\
-0,23 \\
-0,25\end{array}$ \\
\hline & 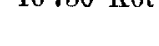 & & Mittel & $\therefore$ & 4,72 & $-0,22$ \\
\hline
\end{tabular}

Von der ersten Periode wurden die drei ersten Tage nicht mitgerechnet. Die Bilanz zeigt das überraschende Resultat, dass die Total-N-Ausgabe in der zweiten Periode sogar geringer ist als in den beiden anderen. Vielleicht ist dies der geringeren N-Ausgabe im Kot beizumessen.

Die Mischung leistet für die Erbaltung des Körperbestandes mindestens so viel wie das Kasein, und es ist deshalb. wenigstens äusserst wahrscheinlich, dass bei etwas grösseren absoluten Mengen der Gelatinemischung sich die negative Bilanz ebensogut wie durch etwas grössere Gaben von Kasein hätte beseitigen lassen.

Gleichzeitig sollte beim kleinen Hund die Hälfte des Kaseins durch kombinierte Leimgabe ersetzt werden.

Er sollte bekommen:

$$
\begin{aligned}
& 232 \mathrm{ccm} \text { Milch . . . 1,25 g N; } 150 \text { Kal., } \\
& 9,82 \text { g Plasmon . . } 1,25 \mathrm{~g} \mathrm{~N} ; 41 \text { " } \\
& 35 \text { "Fett . . " } 332 \text { " } \\
& 10 \text { "Stärke . . . } 41 \text { " } \\
& \begin{array}{lr}
60 \text { „Zucker . . . } & 234, \text { " } \\
\hline \text { Im ganzen } & 2,5 \text { g N } ; 798 \mathrm{Kal}
\end{array}
\end{aligned}
$$


oder, da das Gewicht am 16. Januar $8320 \mathrm{~g}$ betrug, pro Kilogramm $96 \mathrm{Kal}$. und $0,3 \mathrm{~g} \mathrm{~N}$.

Von den 2,5 $\mathrm{g} \mathrm{N}$ sollte er $1,25 \mathrm{~g} \mathrm{~N}$ als Leim- $\mathrm{N}$ bekommen.

Also

$$
0,646 \mathrm{~g} \text { Tyrosin }=0,05 \mathrm{~g} \mathrm{~N} \text {, }
$$

und

$$
\begin{aligned}
& 0,226 " \text { Tryptophan }=0,031 " \mathrm{~N}, \\
& 7,73 \text { "Gelatine }=1,17 \% \mathrm{~N} .
\end{aligned}
$$

Die übrige Nahrung blieb dieselbe, die Anzahl der Kalorien war dieselbe wie in der ersten Periode.

Schon vom zweiten Tag der Hauptperiode an berührte das Tier seine Nahrung nicht mehr, so dass sie bis zum Ende des Versuchs mittelst Schlundsonde gegeben werden musste. Um dem Brechreiz entgegenzuwirken, wurde der Hund nach der Fütterung eine Zeitlang mit erhobenem Kopf hin und her geführt. Es gelang so, Erbrechen zu vermeiden. Um das Gemisch dünnflüssiger zu machen, wurden statt der $10 \mathrm{~g}$ Stärke $10 \mathrm{~g}$ Rohrzucker gegeben, was für die Berechnung der Kalorien nur einen Minimalunterschied ausmachte.

Tabelle III. Kleiner Hund.

$1 / 2$ Leim-N, $4 \%$ Tyrosin, $21 / 2 \%$ Tryptophar.

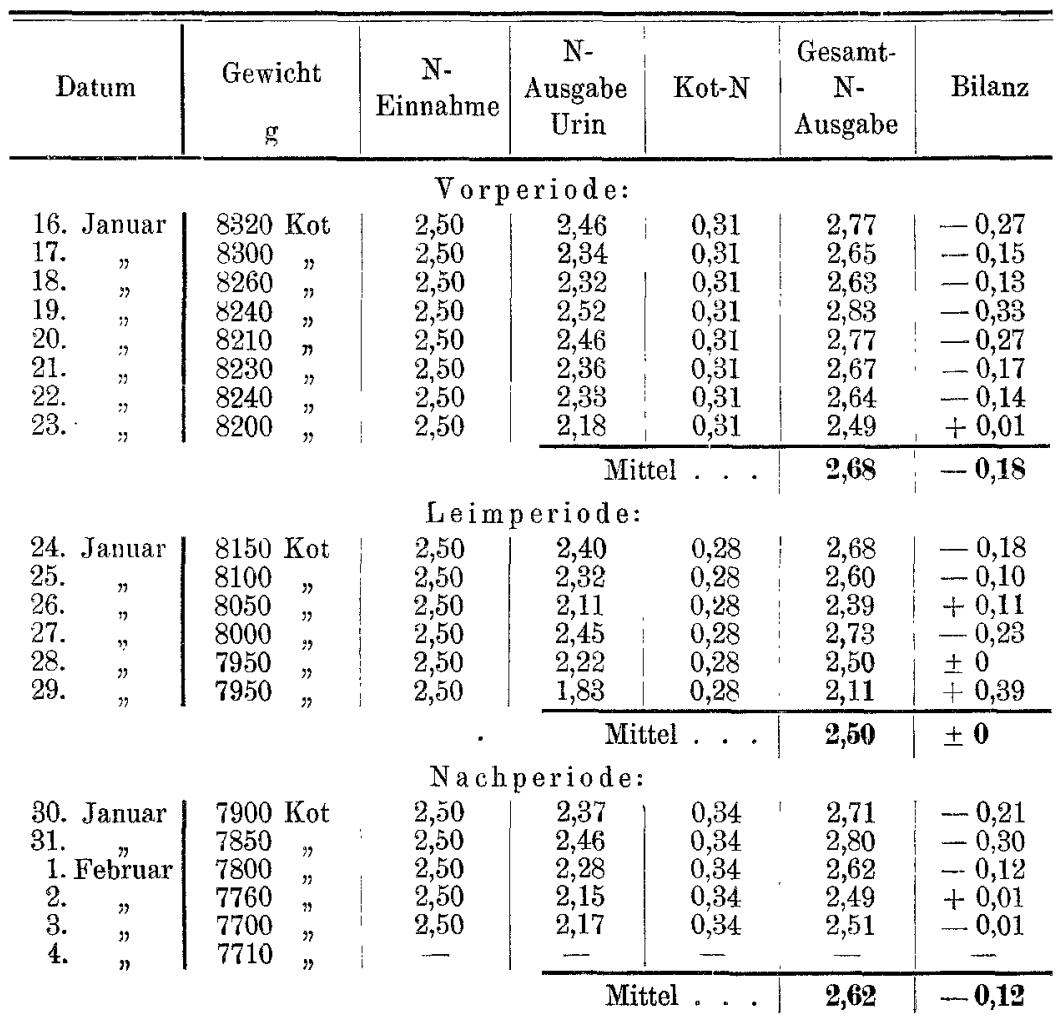


Da die N-Ausgabe gleich rom 16. Januar an gleichmässig war, so wurde in der Vorperiode von diesem Tage an gerechnet.

Hier spricht die N-Bilanz sogar zugunsten der zweiten Periode; trotz der Sondenfütterung wurde die Kost gut resorbiert. Die N-Zahl für Kot ist in der zweiten Periode etwas geringer als in den beiden anderen.

Durch die bisherigen Versuche ist also bewiesen, dass die Vollwertigkeit des Leimes als Ersatz des Eiweisses, welche ihre Grenze findet, wenn $1 / 5$ des Stickstoffs in Form von Leim gegeben wird, durch Zugabe von Tyrosin und Tryptophan bis auf die Hälfte des Gesamtstickstoffs erhöht werden kann. Es war nun zu untersuchen, ob die Vertretbarkeit noch weiter gehe.

Im Leim ist weniger Schwefel enthalten als im Eiweiss. So hat Michel ${ }^{1}$ ) im Serum-Albumin 1,10\% Schwefel gefunden, während die höchste bisher (von Chittenden) gefundene Schwefelzahl im Leim $0,75 \%$ beträgt. Da der Schwefel wohl hauptsächlich auf das schwefelhaltige Cystin zu rechnen ist, so beschloss ich bei einem weiteren Versuche, wobei alles Eiweiss durch Leim ersetzt werden sollte, $2 \%$ des Leim-N durch Cystin-N zu ersetzen, so dass also nur $93 \%$ Leim-N, 4\% Tyrosin-N, $2 \%$ Cystin-N und weniger Tryptophan- $\mathrm{N}$ als früher, nur $1 \%$ gegeben werden sollte.

Cystin stellte ich nach einem mir von Dr. Abderhalden mitgeteilten einfachen Verfahren dar; $300 \mathrm{~g}$ Menschenhaare wurden mit einem Liter rauchender Salzsäure sechs Stunden am Rückflusskühler gekocht, die Lösung noch warm mit Natronlauge alkalisch gemacht, filtriert und das Filtrat in den Eisschrank gestellt.

Von dem kristallisierten Tyrosin und etwaigem Kochsalz wird abfiltriert, das Filtrat wird mit Eisessig angesänert. Es fällt dann reichlich Cystin aus, dasselbe wird in heisser Ammoniaklösung wieder gelöst und durch Eisessig wieder ausgefällt.

Schwierigkeiten musste das Einführen von grossen Mengen Leim bereiten, da die Tiere durch die verschiedenen Stoffwechselversuche. einen Widerwillen gegen Leim bekommen hatten. Andererseits sind so gut fressende und ausdauernde Hunde schwer zu finden. Es wurde deshalb beschlossen, nach einer achttägigen Pause beide Hunde hungern zu lassen. Der grosse Hund sollte von seinem Gewicht $17140 \mathrm{~g}$ bis auf $15 \mathrm{~kg}$ zurückgehen, der kleine Hund von $9080 \mathrm{~g}$ auf $7 \mathrm{~kg}$. Damit sollte zweierlei bezweckt werden:

1) Würzburger physik. medizin. Gesellsch. N. F. Bd. 117. 1895. 
1. sollte die für das Stickstoffgleichgewicht nötige N-Einfuhr entsprechend dem geringeren Körpergewicht verringert werden und damit auch die Leimmenge und

* 2. sollten die Tiere für die unangenehme Kost durch Hunger vorbereitet werden. Der Versuch sollte gleich mit der Leimperiode beginnen, nach acht Tagen die Eiweissperiode sich daran anschliessen.

Beim grossen Hund betrug die N-Ausgabe vom 15. bis 17. Februar:

beim kleinen Hund:

$$
3,14 \mathrm{~g}, \quad 3,20 \mathrm{~g}, \quad 3,16 \mathrm{~g} \text {; }
$$

$$
1,68 \mathrm{~g}, \quad 1,60 \mathrm{~g}, \quad 1,74 \mathrm{~g} .
$$

Am 17. Februar wurde der Kot mit Kieselsäure, die mit 50 bezw. $30 \mathrm{~g}$ Fett vermengt war, abgegrenzt.

An 18. Februar begann der Versuch. Der grosse Hund bekam pro Kiliogramm $861 / 2 \mathrm{Kal}$. und im ganzen $5,61 \mathrm{~g} \mathrm{~N}$.

Der kleine Hund pro Kilogramm $92 \mathrm{Kal}$, und im ganzen 3,5 g N. Nachdem der grosse Hund die ersten fünf Tage gut gefressen hatte, fing er am sechsten Tage an zu brechen, so dass der Versuch aufgegeben werden musste. Die $\mathrm{N}$-Ausgaben im Gesamturin waren vom 18. bis 22. Februar:

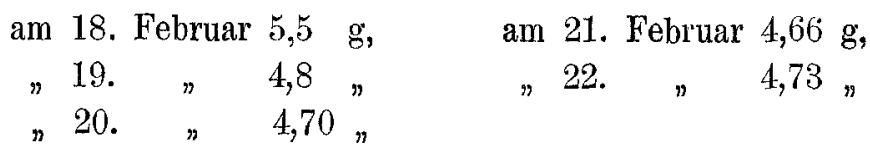

Zahlen, die entschieden die Tendenz zur geringeren $\mathrm{N}$-Ausscheidung zeigen und wahrscheinlich samt 0,4 bis $0,5 \mathrm{~g}$ Kot- $\mathrm{N} 5,16 \mathrm{~g} \mathrm{~N}$ - Einnahme kaum überschritten hätten.

Wenn auch die Vergleichsperiode fehlt, so wissen wir doch aus zahlreichen Versuchen, auch aus den von mir gemachten, dass dieser Hund, wenn er den Leim ohne die Zusätze bekommen hätte, erheblichen Stickstoffverlust erlitten, aller Voraussicht nach eine negative Bilanz in Höhe von ca. $-3 \mathrm{~g} \mathrm{~N}$ pro Tag aufgewiesen hätte. Wenn wir in unserem Falle annehmen, dass er die höchste früher gefundene Stickstoffausscheidung im Kot von $0,5 \mathrm{~g}$ auch in diesem Ealle gehabt hätte, so würden sich die täglichen N-Ausgaben und die Bilanz wie folgt stellen:

$\begin{array}{llll} & & \text { N-Ausgabe } & \text { N-Bilanz } \\ \text { 18. } & \text { Februar } & 6,0 & -0,39, \\ \text { 19. } " & 5,3 & +0,31, \\ \text { 20. } " & 5,2 & +0,41, \\ 21 . & & 5,16 & +0,45, \\ 22 . & & 5,23 & +0,38,\end{array}$


Es scheint also in der Tat, dass die von mir gewählte Mischung einen vollwertigen Ersatz des Eiweisses darstellt.

Der kleine Hund musste gleich mit der Scblundsonde gefüttert werden, brach am zweiten Tage alles aus; das Erbrochene wurde aufgefangen und ihm nach einigen Stunden wieder mit der Schlundsonde gegeben. Als er wieder brach, wurde der Versuch aufgegeben. Der kleine Hund starb am 29. Februar; die Sektion ergab nur allgemeine Abmagerung und ein schlaffes Herz. Der grosse Hund starb am 9. März an einer linksseitigen Lungenentzündung, welche zur Zeit unter den Hunden des Laboratoriums mehrfach Opfer forderte. Der Urin war dauernd eiweissfrei geblieben, die Nieren waren nicht erkrankt. Die Befunde gaben keinen Anhalt dafür, dass die verabreichten Spaltungsprodukte toxische Wirkungen besässen.

Es schien nach diesen Erfahrungen schwierig, wenn nicht unmöglich, Hunden eine den ganzen Stickstoffbedarf deckende Leimmenge einzuführen. Andererseits liess doch der rudimentäre Versuch am grossen Hunde kaum noch einen $Z$ weifel, dass es möglich sein werde, dem Leim durch Beigabe der ihm fehlenden Bauelemente des Eiweisses den vollen Nährwert von Eiweiss zu geben. So beschloss ich denn, den Stoffwechselversuch an mir selbst auszuführen.

\section{Selhstversuch.}

Es handelte sich um einen sehr muskulösen Mann mit geringem Fettpolster, Gewicht $80690 \mathrm{~g}$. Vor dem Versuche wurde der N-Gehalt des Gesamturins von 24 Stunden bestimmt, er betrug $14,6 \mathrm{~g} \mathrm{~N}$. Die Nahrung sollte pro Kilogramm ca. 40 Kal. und $0,2 \mathrm{~g} \mathrm{~N}$ liefern. Als einzige stickstoffhaltige Nahrung sollte das Plasmon dienen, so dass es sich um den alleinigen Ersatz von Kasein-N durch Leim-N handelte; in der Stärke, in der Butter, im Tee und Wein sind so geringe Mengen $\mathrm{N}$ enthalten, dass man sie vernachlässigen kann, da sie entfernt nicht ausreichen, den Eiweissbedarf zu decken, wenn man auch dem Leim extremste eiweisssparende Wirkung zuschreiben wollte. Bei den Hunden hatte der Zusatz von Stärke der abführenden Wirkung des Leims entgegengewirkt. Da viel Butter aufzunehmen war, so war zu befürchten, dass trotz der miteingeführten Stärke sich Durchfall einstellen würde. Deshalb, und weil es sich zugleich um eine Person handelte, die an Alkoholgenuss gewöhnt war, wurde ein stark gerbsäurehaltiger sizilianischer Rotwein "Etna" bezogen, 
dessen Reinheit und gleiche Zusanmensetzung in jeder Flasche garantiert wurde. Während der Versuchszeit hat der feurige Wein nicht wenig dazu beigetragen, die Verdauung anzuregen und den Brechreiz zu bekämpfen.

Das Plasmon wurde mit Salz und Zucker, Stärke, Butter und etwas Himbeersaft zu einer Art Kuchen zusammengebacken und dazu aus Himbeersaft, etwas Wein und Wasser eine Sauce bereitet. Zum Frühstück wurde aus $1 \mathrm{~g}$ Tee und $10 \mathrm{~g}$ Zucker ein leicht anregendes Getränk bereitet. Der Plasmonkuchen wurde mittags und abends genommen, der Wein und beliebig Wasser wurden im Laufe des Tages getrunken.

Schwieriger war es, die grossen Mengen Gelatine geniessbar zu machen. Denn die erkaltete Masse schmeckte mit der Stärke gemischt widerlich klebrig wie Leim. Deshalb wurde die Gelatine mit der Butter zusammen erst erwärmt und flüssig gemacht, in der Flüssigkeit die Stärke, der Zucker, die abgewogene Menge Tyrosin, Cystin und Tryptophan, etwas Himbeersaft und reichlich Wein verrührt und noch warm getrunken. Diesmal wurde eine rot gefärbte, auch wieder vollkommen eiweissfreie Gelatine verwandt. Der Farbstoff wurde chemisch als neutrales Fuchsin identifiziert. Dieser rote Farbstoff ging auch auf den Kot über, wie es sich bei dem vorigen Tierversuch gezeigt hatte. Dadurch grenzte sich der Kot deutlich von der anderen Periode ab. Vor Beginn des Versuchs und nach der dritten Periode wurde der Kot durch hohes Klistier abgegrenzt. Die kotfärbende Wirkung des neutralen Fuchsins wurde von mir späterhin geprüft und dabei gefunden, dass etwa $1 / 2 \mathrm{~g}$ des etwas bitter schmeckenden Fuchsins genügt, um den Kot deutlich rot zu färben und somit abzugrenzen.

Dieses Fuchsin muss als stickstoffhaltiger Stoff mit berücksichtigt werden. Da nach der Kotfärbung zu sehliessen weniger als $0,5 \mathrm{~g}$ des Farbstoffes eingeführt wurden, so können, da das Fuchsin, dessen Hauptbestandteil das Rosanilin, das Chlorhydrat des Anhydro- $\mathrm{p}_{3}$ triamidodiphenyl-m-tolylcarbinols: $\mathrm{C}_{20} \mathrm{H}_{19} \mathrm{~N}_{3} \cdot \mathrm{HCl}+4 \mathrm{H}_{2} \mathrm{O}$ einen Stickstoffgehalt von $10,23 \%$ hat, höchstens $0,05 \mathrm{~g} \mathrm{~N}$ dem Fuchsin angehören. Da dieser Stickstoff für die Eiweisssynthese wohl schwerlich etwas bedeutet, so wird durch den Ersatz von $\check{c}$ cg Leim- $\mathrm{N}$ durch Fuchsin- $\mathrm{N}$ das Resultat nur prägnanter.

In der Vor- und Nachperiode wurde an Nahrung eingenommen: 
$110 \mathrm{~g}$ Plasmon (bezw. vom dritten Tage an $120 \mathrm{~g}$ )

$12,72 \% \mathrm{~N}, 0,5 \%$ Fett und 4,1 Kal. pro Gramm

135 g Rohrzucker . . . . . . . . . 3,9 " " " 100 "Stärke $0,4 \% \mathrm{~N}$. . . . . . . 4,1 " " " 150 "Butter (vom dritten Tage an $175 \mathrm{~g}$ ), deren Fettgehalt entsprechend dem gewöhnlichen Befund $87 \%$ ig angenommen wurde, $0,18 \% \mathrm{~N}$ und 8,03 Kal. pro Gramm.

$600 \mathrm{~g}$ Wein $0,033 \% \mathrm{~N} ; 10,36 \%$ Alkohol. Vom Alkohol müssen $2 \%$ als unverbrannt verloren gehend berücksichtigt werden. Ich rechne deshalb pro Gramm 7,0 Kal.

$70 \mathrm{~g}$ Himbeersaft, davon $60 \%$ Trockensubstanz $=$ Traubenzucker,

1 "Saft $=2,34$ Kal.

1 , Tee (Infus) 1,02\% N, also

in Plasmon $13,99 \mathrm{~g} \mathrm{~N}$ resp. $15,26 \mathrm{~g} \mathrm{~N}$; $451 \mathrm{Kal}$. resp. $492 \mathrm{Kal}$.

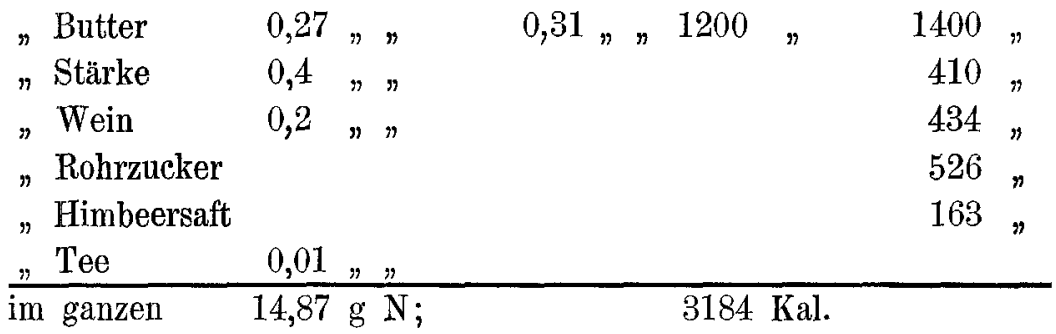

bezw. vom dritten Tage an $16,1 \mathrm{~g} \mathrm{~N}$; $3425 \mathrm{Kal}$; pro Kilogramm $0,18 \mathrm{~g} \mathrm{~N}$ bezw. $0,2 \mathrm{~g} \mathrm{~N}$ und 39 bezw. $42 \mathrm{Kal}$.

Am zweiten Versuchstage hatte das Gewicht um $720 \mathrm{~g}$ abgenommen, am dritten Tage $980 \mathrm{~g}$, deshalb wurde die Nahrungseinnahme erhöht durch Mehrgabe von $10 \mathrm{~g}$ Plasmon und $25 \mathrm{~g}$ Butter.

In der Hauptperiode traten an die Stelle von $120 \mathrm{~g}$ Plasmon $=15,264 \mathrm{~g} \mathrm{~N}$, dieselbe Menge $\mathrm{N}$ in der Weise, dass $93 \%$ ersetzt wurden durch Gelatine-N, $4 \%$ durch Tyrosin-N, $2 \%$ durch Cystin-N und $1 \%$ durch Tryptophan-N. Die angewandte Gelatine enthielt $14,28 \% \mathrm{~N}$. Somit wurden ausgerechnet $99,4 \mathrm{~g}$ Gelatine $=14,19 \mathrm{~g} \mathrm{~N}$, $7,89 \mathrm{~g}$ Tyrosin $=0,6104 \mathrm{~g} \mathrm{~N}, 1,11 \mathrm{~g}$ Tryptophan $=0,1526 \mathrm{~g} \mathrm{~N}$.

Cystin, Dithio - diamido - dimilchsäure

$\mathrm{CH}_{2}-\mathrm{S}-\mathrm{S}-\underset{\mathrm{CH}}{\mathrm{CH}_{2}}$
$\mathrm{CH} \cdot \mathrm{NH}_{2}$
$\mathrm{COOH}$
$\mathrm{COOH}$

enthält $11,66 \% \mathrm{~N}$, die Kalorienzahl ist unbekannt; davon wurden berechnet $2,61 \mathrm{~g}=0,3052 \mathrm{~g} \mathrm{~N}$. 
Die übrige Nahrung war dieselbe wie in der Vorperiode. Die Kalorienzahl kommt der in der Vorperiode ziemlich gleich.

An die Hauptperiode wurden noch drei Tage Nachperiode angeschlossen. Die N-Ein- und Ausgaben sind aus beiliegender Tabelle zu ersehen:

Tabelle IV. Selbstversuch.

Ersatz von allem Kasein-N durch Leim-N.

\begin{tabular}{|c|c|c|c|c|c|c|c|c|}
\hline Datum & $\begin{array}{c}\text { Gewicht } \\
\mathrm{g}\end{array}$ & $\begin{array}{l}\text { Flüssig- } \\
\text { keits- } \\
\text { auf- } \\
\text { nahme }\end{array}$ & $\begin{array}{c}\text { Urin- } \\
\text { menge }\end{array}$ & $\begin{array}{c}\mathrm{N}- \\
\text { Ein- } \\
\text { nahme }\end{array}$ & $\begin{array}{c}\text { Urin- } \\
\mathrm{N}\end{array}$ & $\begin{array}{l}\text { Kot- } \\
\mathrm{N}\end{array}$ & $\begin{array}{l}\text { Total- } \\
\text { N- } \\
\text { Aus- } \\
\text { gabe }\end{array}$ & Bilanz \\
\hline 6. April & 80690 Kot & 2540 & 1730 & 14,87 & 14,95 & 1,74 & 16,69 & $-1,82$ \\
\hline \multicolumn{9}{|c|}{ Vorperiode: } \\
\hline $\begin{array}{l}\text { 7. April } \\
8 . \quad " \\
9 . \quad "\end{array}$ & $\begin{array}{l}79970 \text { Kot } \\
79000 \quad " \\
79640 \%\end{array}$ & $\begin{array}{l}2010 \\
3565 \\
2810\end{array}$ & \begin{tabular}{|l}
14256 \\
1425 \\
2470
\end{tabular} & $\begin{array}{l}14,87 \\
16,18 \\
16,18 \\
\end{array}$ & $\begin{array}{l}14,43 \\
15,04 \\
15,47 \\
\end{array}$ & $\begin{array}{l}1,74 \\
1,74 \\
1,74 \\
\end{array}$ & $\begin{array}{l}16,17 \\
16,78 \\
17,21 \\
\end{array}$ & $\begin{array}{r}-1,30 \\
-0,60 \\
-1,03 \\
\end{array}$ \\
\hline
\end{tabular}

Leimperiode:

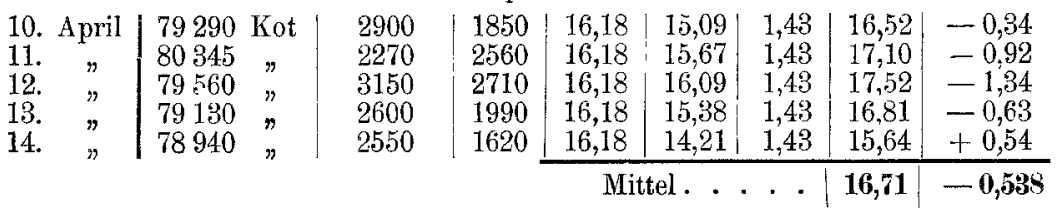

Nachperiode:

\begin{tabular}{|c|c|c|c|c|c|c|c|c|}
\hline \multirow{4}{*}{$\begin{array}{l}\text { 15. April } \\
16 . " ~\end{array}$} & \multirow{4}{*}{$\begin{array}{l}78825 \text { Kot } \\
79015 " ~ \\
78920 " \\
78810 "\end{array}$} & 2570 & | 2430 & 16,18 & 13,81 & 1,75 & 15,56 & $+0,62$ \\
\hline & & 2400 & 2135 & 16,18 & 14,47 & 1,75 & 16,22 & $-0,04$ \\
\hline & & 2700 & 2560 & 16,18 & 14,79 & 1,75 & 16,54 & $-0,36$ \\
\hline & & & & & & & 16,10 & $+0,07$ \\
\hline
\end{tabular}

Der erste Tag ist ausser Betracht zu lassen, weil hier noch eine Nachwirkung der früheren Kost anzunehmen ist. In den ersten Tagen bestanden grosse Abgeschlagenheit, schlechtes Aussehen, jedoch kein Fieber. Der Urin war sauer und eiweissfrei. Bei Betrachtung der Gewichtszahlen der einzelnen Tage fallen die grossen Schwankungen auf. Gewogen wurde um dieselbe Zeit unter denselben Bedingungen. Tatsächlich entsprach der starken Gewichtsabnahme der ersten Tage nicht ein grosser Stickstoffverlust; die Gewichtsabnahme und auch die Schwankungen sind wohl nur auf Wasserverlust zu beziehen, ein Beweis, wie vorsichtig Wägungen bei veränderter Kost aufgefasst werden müssen. Während des ganzen Versuches bestand grosse Mattigkeit und Unlust zur Arbeit, auch die Stimmung war etwas deprimiert; die Kalorienzufuhr war reichlich, wenn sie auch anscheinend den Bedarf nicht ganz deckte und der N-Verlust nicht 
sehr gross, deshalb ist die grosse Mattigkeit wahrscheinlich auf das Fehlen von genügend Extraktstoffen, die ja eine Nahrung schmackhaft machen und anregend auf das Nervensystem wirken, auf die mit Widerwillen genossene Kost zu beziehen. Vielleicht spielen auch gewisse Toxinwirkungen eine Rolle.

Die Vergleichung der mittleren $\mathrm{N}$-Ausgaben in den drei Perioden zeigt eine Tendenz zur Verringerung derselben. Die Hauptperiode steht auch in dieser Hinsicht in der Mitte. Während dieser war übrigens das Befinden noch relativ am besten. Ganz auffallend ist die geringere Kot-N-Zahl in der zweiten Periode. Sie beweist, dass der Leim- $\mathrm{N}$ besser ausgenutzt wurde als das leicht lösliche Caseinnatrium.

Selbst wenn man die günstige Bilanz der zweiten Periode im Vergleich zur ersten auf die geringere Kot-N-Zahl beziehen muss, so scheint doch der Zusatz der erwähnten aromatischen Aminosäuren den Leim dem Eiweiss fast gleichwertig zu machen. Vergleicht man mit dem Resultat der Hauptperiode, wo fast sämtlicher Eiweiss-N bis auf die in Butter und Stärke enthaltenen Spuren durch Leimstickstoff ersetzt wurde, die Tabelle I, kleiner Hund, wo schon durch den Ersatz von ein Drittel Eiweiss-N durch Leim-N ein Stickstoffverlust entstand, der fast drei Viertel des Leim-N entsprach, so leuchtet ohne weiteres ein, dass der mit den erwäbnten aromatischen Aminosäuren versetzte Lein physiologisch dem Eiweiss nahekommt, ja ihm vielleicht vollkommen gleichwertig ist. Ob das von mir angewandte Aminosäurengemisch gerade das günstigste ist, ob nicht andere Zusätze zum Leim noch günstiger wirken würden, das zu untersuchen muss der Zukunft vorbehalten bleiben.

In Urin konnte keine der drei genossenen Aminosäuren nachgewiesen werden.

Das Tyrosin konnte weder mikroskopisch durch die typischen Nadeln, noch durch die Proben von Piria, Denigès und Wurster nachgewiesen werden.

Das Tryptophan konnte im Urin weder mit der Adamkiewiezschen Glyoxylsäurereaktion noch durch die Tryptophanreaktion (Rotfärbung auf Zusatz von Bromwasser), noch durch die Pyrrolreaktion nachgewiesen werden. Die Proben auf Indol und Skatol fielen alle negativ aus. Auch der Nachweis von Indoxylschwefelsäure gelang nicht. Nach den neuesten Untersuchungen über Tryptophan entspricht dies dem allgemeinen Befund ${ }^{1}$ ).

1) Zeitschr. fo chem. Phys. u. Path. Bd.4 S. 171. Ellinger u. Gentzen. 
Im Harnsediment fanden sich sechsseitige Tafeln, die zuerst für Cystin gehalten wurden. Bei Erwärmung derselben mit Ammoniak und nachherigem Zusatz von Essigsäure erfolgte aber keine Kristallisation mehr. Es handelte sich also um Harnsäurekristalle. Um das Cystin chemisch nachzuweisen, wurde 1 Liter Harn mit $10 \mathrm{ccm}$ Benzoylchlorid und überschüssiger Natronlauge bis zum Verschwinden des Benzoylchloridgeruchs geschüttelt, vom Niederschlag wurde abfiltriert, das Filtrat wurde mit Schwefelsäure angesäuert und mit Äther ausgeschüttelt. Nach Verdampfung des Äthers wurde der Rückstand mit Natronlauge und Bleiacetat erwärmt, es bildete sich aber kein Schwefelblei. Somit konnte Benzoylcystin nicht isoliert werden, folglich enthielt der Harn kein Cystin. Dagegen entstand beim Kochen des Harns mit Natronlauge und Bleiacetat Schwefelblei.

Dieser bleischwärzende Schwefel wurde nach K. A. H. Mörner ${ }^{1}$ ) in folgender Weise quantitativ bestimmt: 1 Liter Urin wurde mit $50 \mathrm{~g} \mathrm{NaOH}$ und $10 \mathrm{~g}$ Bleiacetat zwölf Stunden lang gekocht. Das ausgeschiedene Schwefelblei wurde im Goochtiegel gesammelt, mit verdünnter Natronlauge ausgewaschen, nun nach Zusatz von Salpetersäure mit Bromwasser oxydiert. Die Lösung wurde im Wasserbad eingedampft, der Rückstand mit Natriumkarbonat und etwas Wasser aufgenommen, in einen Silbertiegel überführt und über einer Weingeistflamme eingetrocknet. Jetzt wurde mit Wasser ausgelaugt, das Ungelöste noch einmal mit Sodalösung erwärmt und mit Wasser ausgewaschen. Das Filtrat wurde mit Bromwasser versetzt, mit Salzsäure übersättigt, auf dem Wasserbade eingetrocknet. Der Rückstand wurde mit Salzsäure und Wasser aufgenommen, filtriert; nach genügender Verdünnung mit Wasser wurde die Schwefelsäure als Baryumsulfat bestimmt.

Der nach dieser Methode bestimmte bleischwärzende Schwefel differierte in zwei Tagen erheblich. Am zweiten Tage der Hauptperiode wurden $0,0203 \mathrm{~g} \mathbf{S}$ gefunden, am dritten Tage 0,0138 . Vielleicht wurde an den einzelnen Versuchstagen mehr oder weniger S zu Schwefelsäure weiter oxydiert.

An normalen Tagen wurde kein bleischwärzender Schwefel in meinem Harn gefunden.

Sadik off ${ }^{2}$ ) fand bei seinen Untersuchungen über tierische Leimstoffe bei Kjeldahl-Bestimmungen den Stickstoffgehalt der

1) Zeitschr. f. physiol. Chemie Bd. 34 S. 207.

2) Zeitschr. f. physiol. Chemie Bd. 39 S. 396; Bd. 41 S. 15. 
Gelatine niedriger als nach der Methode von Dumas. So fand er nach Kjeldahl $\mathrm{N}=17,35$, nach Dumas $\mathrm{N}=17,82$; ferner nach Kjeldahl $\mathrm{N}=17,46$, nach Dumas $\mathrm{N}=17,87$. Weiterbin nach Kjeldahl 17,43, nach Dumas 18,08 und Kjeldahl 17,47, Dumas 17,67 usw. Es wurde also nach Kjeldahl meist etwa $1 / 2 \% \mathrm{~N} \mathrm{zu}$ wenig gefunden. Der Autor schloss auf mit $20 \mathrm{cem}$ Schwefelsäure, $1 \mathrm{~g}$ Kupfersulfat und $10 \mathrm{~g}$ Kaliumsulfat, meist zwei Stunden lang.

Ich habe stets über drei Stunden aufgeschlossen, und zwar mit Schwefelsäure nebst einem kleinen Tropfen Quecksilber; feste Stoffe liess ich im Aufschlusskolben mit Wasser angesetzt einige Zeit stehen, damit nicht durch die konzentrierte Schwefelsäure eine plötzliche Verkohlung und Erhitzung, ein Entweichen von flüchtigen N-Verbindungen wie $\mathrm{NO}_{2}$ eintrat. Bei zwei Qualitäten Gelatine habe ich den Stickstoff nach Dumas und Kjeldahl bestimmt. Die Verbrennungen nach Dumas, die im ersten chemischen Institut gemacht wurden, gingen sehr langsam vor sich und dauerten ca. vier Stunden, auch nachdem anstatt Kupferoxyd Bleichromat genommen war. Die Gelatine ist auf dem Platinblech schwer zu veraschen; kleine Stücke verbrennen über der stärksten Gebläseflamme erst nach zwei Minuten vollständig.

Folgende Zahlen wurden gefunden:

$\mathrm{N}$ nach Kjeldahl: 15,129\%, 15,131\% und 15,130\%, Dumas: $14,88 \%, \quad 14,78 \%$.

Ferner bei einer weiteren besonders langwierigen Verbrennung: Nach Dumas: $14,28 \% \mathrm{~N}$, nach Kjeldahl: 14,28\% $\mathrm{N}$ im Mittel.

Letztere Qualität Gelatine wurde auch nach der Kjeldahlmethode mit $\mathrm{Kupfersulfat}$ aufgeschlossen.

Es wurden Zahlen gefunden : 13,96 g, 14,12 g, 14,20 g, 13,91 g N, die nicht nur unter sich mehr als zulässig differierten, sondern immer zu wenig Stickstoff ergaben, ein Beweis, dass die Methode mit Kupfersulfat bei so schwer verbrennbaren Körpern nicht ausreicht und die katalytische Wirkung des Quecksilbers beim Aufschliessen mit Schwefelsäure eine wichtige Rolle spielt.

Herrn Prof. Zuntz möchte ich auch an dieser Stelle für die mannigfache Unterstützung und Beratung, die er mir während der ganzen Arbeit zuteil werden liess, ergebenst danken. 